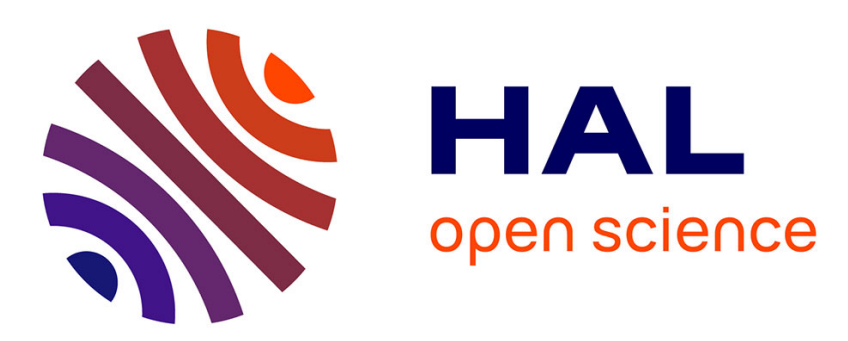

\title{
Discrete wavelet for multifractal texture classification: application to medical ultrasound imaging
}

Meriem Djeddi, Abdeldjalil Ouahabi, Hadj Batatia, Adrian Basarab, Denis Kouamé

\section{- To cite this version:}

Meriem Djeddi, Abdeldjalil Ouahabi, Hadj Batatia, Adrian Basarab, Denis Kouamé. Discrete wavelet for multifractal texture classification: application to medical ultrasound imaging. IEEE International Conference on Image Processing (ICIP 2010), IEEE Signal Processing Society, Sep 2010, Hong Kong, China. pp.637-640, 10.1109/ICIP.2010.5650017 . hal-03149774

\section{HAL Id: hal-03149774 \\ https://hal.science/hal-03149774}

Submitted on 26 Feb 2021

HAL is a multi-disciplinary open access archive for the deposit and dissemination of scientific research documents, whether they are published or not. The documents may come from teaching and research institutions in France or abroad, or from public or private research centers.
L'archive ouverte pluridisciplinaire HAL, est destinée au dépôt et à la diffusion de documents scientifiques de niveau recherche, publiés ou non, émanant des établissements d'enseignement et de recherche français ou étrangers, des laboratoires publics ou privés. 


\title{
DISCRETE WAVELET FOR MULTIFRACTAL TEXTURE CLASSIFICATION: APPLICATION TO MEDICAL ULTRASOUND IMAGING
}

\author{
Djeddi Meriem ${ }^{1}$, Ouahabi Abdeldjalil ${ }^{2}$, Batatia Hadj ${ }^{1}$, Basarab Adrian ${ }^{1}$, and Kouamé Denis ${ }^{1}$ \\ ${ }^{1}$ Université de Toulouse, IRIT UMR CNRS 5505, ${ }^{2}$ Université de Tours, Signal \& image Group
}

\begin{abstract}
This paper deals with multifractal characterization of skin cancer in ultrasound images. The proposed method establishes a multifractal analysis framework of such images based on a new multiresolution indicator, called the maximum wavelet coefficient, derived from the wavelet leaders.

Two main contributions are brought up: first, it proposes a method for the estimation of multifractal features. Second, it reveals the potential of multifractal features to characterize skin melanoma.

In order to study the efficiency of our maximum coefficient estimator, we compare its results on a simulated image against wavelet leaders based estimator. We then apply the approach on various samples from different skin images. Results show that the extracted features make a promising quantitative indicator to distinguish between different tissues.
\end{abstract}

Index Terms - Multifractal analysis, wavelet, ultrasound imaging, melanoma, tissue characterization.

\section{INTRODUCTION}

Melanoma is a serious and increasingly growing form of skin cancer. Its early diagnosis has been recognized as a fundamental issue for public health policy. This requires an accurate and reliable detection procedure that allows dermatologists to distinguish skin tumors from inherently complex skin structures. Biopsy is the gold standard for existing diagnostic approaches, which are mostly invasive and relatively expensive. Ultrasound imaging is a useful, convenient and safe diagnosis modality of skin pathologies. Unfortunately, this type of images suffer from a high speckle noise, due to the interference of back scattered signals. This noise degrades the image quality (in terms of resolution and contrast), and makes the characterization difficult even for experienced clinicians.

A large number of research studies have addressed the identification of pathological tissues in ultrasound images.
The fractality of the speckle is one of the explored approaches.

The idea of studying statistical scaling laws of the speckle in order to extract underlying features is not recent. One way to describe such a law (or self similarity) is to calculate its fractal dimension [1]. Fractal and multifractal analyses have, indeed, been already used to characterize the speckle and segment abnormal regions from ultrasound imaging [2].

Guyot [3] studied the mathematical correlation between a fractional Brownian motion and speckle patterns, in optical imaging and used successfully the diffusion equation to characterize skin psoriasis infections.

In [4], Chen et al. estimate the fractal dimension of an ultrasound image of breast lesions, also, using the fractal Brownian motion and integrate this estimation in a computer aided diagnosis to classify the lesions as benign or malign.

Lee et al. [5] describe the feasibility of selecting a fractal features vector based on M-band wavelet transform, and use it to classify ultrasonic liver images as normal, cirrhosis or heapatoma. They noticed, from experimental results, that the fractal dimension is a determinant parameter for the classifier. In [6], the same authors developed an unsupervised segmentation algorithm based on these multiresolution fractal features vector.

The major limitation of the reviewed methods comes from the systematic monofractal analysis adopted to compute the fractal dimension. This cannot account for the complexity of skin melanoma images.

To date, few studies have investigated the multifractal analysis of ultrasound images. One of the attempts [7] was to detect boundaries of abnormal regions in ultrasound breast images.

In this paper, we investigate the efficiency of multifractal analysis for ultrasound skin tissue characterization. We show that multifractal attributes can separate two populations of tissues. To our knowledge, this is the first attempt to use this technique in ultrasound skin images.

The paper is organized as follows: In section 2, we first review concepts related to multifractal analysis based on hierarchical multiresolution parameters. We then present our maximum wavelet coefficient and the proposed estimator, and briefly discuss its complexity. We study its performance, in section 3, while presenting and discussing 
the experimental results on a set of in vivo ultrasound skin images. Section 4 concludes and outlines perspectives for future investigations.

\section{METHOD}

\subsection{Related work}

The multifractal analysis, considered as a model of scale invariance, is a standard tool to describe the variation of local regularity in a given function or data. This description is made through the singularities spectrum $D(h)$. This spectrum represents the Hausdorff dimension of Holder exponents $h$ in each point [8].

The estimation of $D(h)$ from a single finite observation is an important and difficult practical question, which can be addressed only through the multifractal formalism.

In order to implement such formalism, it is necessary to use multiresolution parameters which link with the properties of regularity. It is usually put in practice using wavelet transform modulus maxima (WTMM) [9] and more recently the wavelet leaders $[8,10]$.

WTMM is based on the skeleton of a continuous wavelet transform in 2D. However, their averred efficiency is hindered by their very high computational cost. The coefficient leaders, derived from discrete 2D wavelet coefficients, try to remedy this problem as explained below.

Let $\left\{X\left(k_{n_{1}}, k_{n_{2}}\right)\right\}_{n_{1}, n_{2}=1}^{N}$ be the analyzed image and $d_{X}^{(m)}\left(\boldsymbol{K}_{j}\right), m=1,2,3$ the coefficients of the 2D discrete wavelet transform [11]; where $\boldsymbol{K}_{j}=\left(j, k_{1}, k_{2}\right)$ are the coordinates of a position $\left(k_{1}, k_{2}\right)$ at the resolution $j$. Let $\lambda\left(\boldsymbol{K}_{j}\right)$ be the dyadic square indexing that represents the index of all discrete coefficients for different scales at $\left(k_{1}, k_{2}\right)$ :

$$
\lambda\left(\boldsymbol{K}_{j}\right)=\left[\left\{k_{1} 2^{j},\left(k_{1}+1\right) 2^{j}\right\},\left\{k_{2} 2^{j},\left(k_{2}+1\right) 2^{j}\right\}\right]
$$

The union of nine such neighbor intervals is denoted as:

We define:

$$
\lambda^{\prime}\left(\boldsymbol{K}_{j}\right)=\mathrm{U}_{n_{1}, n_{2=\{-1,0,1\}}} \lambda\left(\mathrm{j}, \mathrm{k}_{1}+\mathrm{n}_{1}, \mathrm{k}_{2}+\mathrm{n}_{2}\right)
$$

$$
\begin{array}{r}
D_{X}\left(\boldsymbol{K}_{j}\right)=\left\{d_{X}^{(m)}\left(\boldsymbol{P}_{j}\right)\right\} ; \\
m=1,2,3 ; \quad \boldsymbol{P}_{j} \in \lambda^{\prime}\left(\boldsymbol{K}_{j}\right) .
\end{array}
$$

The wavelet leader $[8,10]$ is defined as follows:

$$
L_{X}\left(\boldsymbol{K}_{j}\right)=\operatorname{Max}\left|D_{X}\left(\boldsymbol{K}_{j}\right)\right| .
$$

The basic result underlying the use of wavelet leaders for multifractal analysis is that: If $X$ has Holder exponent $h$ at point $\boldsymbol{K}_{0}$, then it can be approximated by the wavelet leader as [10]:

$$
L_{X}\left(\boldsymbol{K}_{j}\right) \leq 2^{j h\left(\boldsymbol{t}_{0}\right)}, 2^{j} \rightarrow 0, \boldsymbol{t}_{0}=2^{-j} \boldsymbol{K}_{0} .
$$

These coefficients represent monotone increasing quantities through scales. However, despite their good performance in estimating multifractal attributes, they become instable for large statistical moments, leading to significant bias. Their computation time is not as high as WTMM, but still significant.

\subsection{The proposed method}

These limitations have been the main motivating factor for establishing our maximum wavelet coefficient based estimator, described as follows.

Given

$$
\begin{aligned}
& D^{\prime}{ }_{X}\left(\boldsymbol{K}_{j}\right)=\left\{d_{X}^{(m)}\left(\boldsymbol{P}_{j}\right)\right\} ; \\
& m=1,2,3 ; \quad \boldsymbol{P}_{j} \in \lambda\left(\boldsymbol{K}_{j}\right),
\end{aligned}
$$

We define the maximum wavelet coefficient from the wavelet leaders as:

$$
M_{X}\left(\boldsymbol{K}_{j}\right)=\operatorname{Max}\left|D_{X}^{\prime}\left(\boldsymbol{K}_{j}\right)\right| .
$$

Empirically, we have established that $M_{X}\left(\boldsymbol{K}_{j}\right)$ verify [12]:

$$
M_{X}\left(\boldsymbol{K}_{j}\right) \leq 2^{j h\left(\boldsymbol{t}_{0}\right)}, 2^{j} \rightarrow 0, \boldsymbol{t}_{0}=2^{-j} \boldsymbol{K}_{0} .
$$

As a consequence of this property, we define our estimator of Holder exponent in the subsequent manner.

Let $S\left(2^{j}, q\right)$ be the structure functions, representing the spatial average of wavelet coefficients (of q order) at a given scale $2^{j}$ :

$$
S\left(2^{j}, q\right)=\frac{1}{n_{j}} \sum_{k_{1}, k_{2}} M_{X}\left(\boldsymbol{K}_{j}\right)^{q} .
$$

It has been shown that functions $S\left(2^{j}, q\right)$ exhibit power law behavior with respect to the analysis scale $2^{j}$, in the limit of small scales $\left(2^{j} \rightarrow 0\right)[9]:$

$$
S\left(2^{j}, q\right) \cong C_{q} 2^{j \zeta(q)} \text { when } j \rightarrow-\infty ;
$$

Where $C_{q}$ is a constant, and

$$
\zeta(q)=\lim _{2^{j} \rightarrow 0} \inf \frac{\ln s\left(2^{j}, q\right)}{\ln 2^{j}} .
$$

The $\zeta(q)$ are termed the scaling exponents. This power law behavior establishes a clear connection between the concept of scale invariance and multifractal analysis.

Finally, the Holder exponent $h$ and the multifractal spectrum $D(h)$ can be estimated from $\zeta(q)$ using Chhabra et al. [13].

The maximum wavelet coefficients which have been shown to reproduce the behavior of scaling laws estimate entirely $D(h)$ [12] with better efficiency compared to the wavelet leaders. As it has been reported [14], the complexity of the wavelet leaders estimator is $O(n \log n)$ (where $\mathrm{n}$ is the image size), mainly due to the wavelet coefficients computation phase. Our method shares this same preliminary phase and therefore has a similar complexity. 


\section{RESULTS}

\subsection{Numerical simulation}

The performance of the estimation procedure has been analyzed by generating a large number of realizations of a synthetic process. We use Mandelbrot multiplicative cascades with a minimal regularity and log-poisson multipliers (CMC-LP) [15]. These cascades are multifractal and their scale invariance properties are entirely determined by the spectrum of singularities $D(h)[12,15]$.
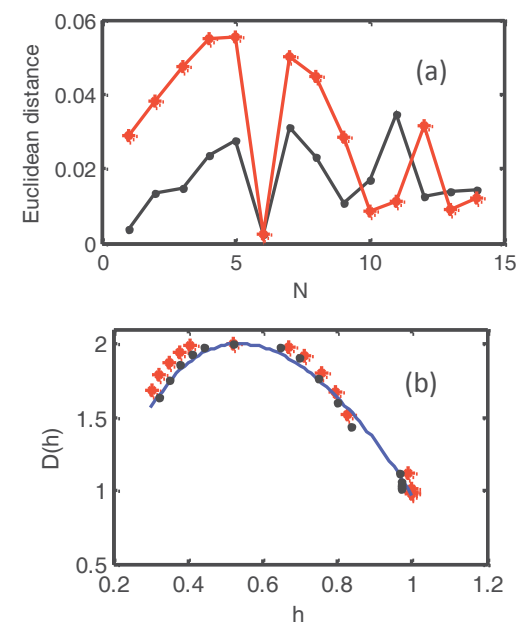

Fig. 1. Estimation performance: a) Evolution of the Euclidian distance between the estimates and the theoretical spectrum, b) Theoretical (blue) vs. maximum coefficient (• black) vs. wavelet leaders $(*$ red) based spectrum.

We evaluated quantitatively the performance of our estimator by estimating the average Euclidean distance between the estimated $\widehat{D}(h)$ and the theoretical spectrum of singularity $D_{T}(h)$. This is defined as the average of the distance between each point $\left(\widehat{h}_{i}, \widehat{D}_{i}\right)$ of $\widehat{D}(h)$ and its nearest neighbor $\left(h_{i}, D_{i}\right)$ on the theoretical spectrum.

$$
d_{E}\left(\widehat{D}(h), D_{T}(h)\right)=\frac{1}{N} \sum_{i=1}^{N} \sqrt{\left(h_{i}-\widehat{h}_{i}\right)^{2}+\left(D_{i}-\widehat{D}_{i}\right)^{2}}
$$

Where $N$ is the number of points of the estimated curve.

\begin{tabular}{|c|c|c|}
\hline & CmaxWT spectra & WL spectra \\
\hline Distance (d) & 0.0473 & 0.0146 \\
\hline
\end{tabular}

Table 1. Estimation of the euclidien distance between the theoretical curve and the estimation ones.

We notice (Table 1) that the multifractal spectrum based on our estimator obtains better fit to the theoretical curve, although more precise for positive statistical moments (Figure1).

\subsection{Application of the multifractal formalism to ultrasound skin images}

As an application, we conducted a preliminary study of the characterization of melanoma using multifractal analysis in ultrasound skin images, and compared it to the fractal approach proposed in [3]. Our preliminary corpus was made of seven skin ultrasound images provided by a clinical study from the hospital of Toulouse, France.

To demonstrate the performance of the proposed method, we applied the analysis to 14 samples (selected by the clinician from the 7 images). We, then, extracted multifractal and fractal features from each sample in order to investigate the ability of each parameter to differentiate normal from pathological tissues.
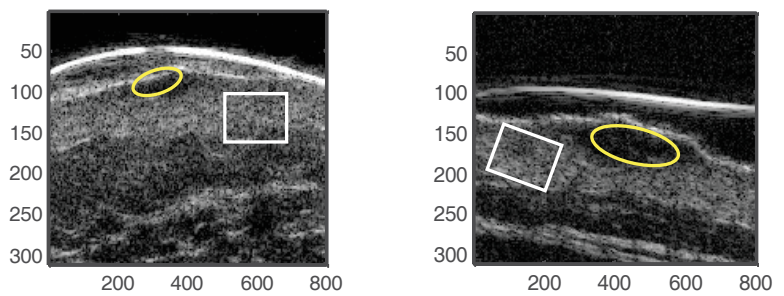

Fig 2. Ultrasound skin images: the yellow ellipse delimits a melanoma and the white square surrounds normal dermis.
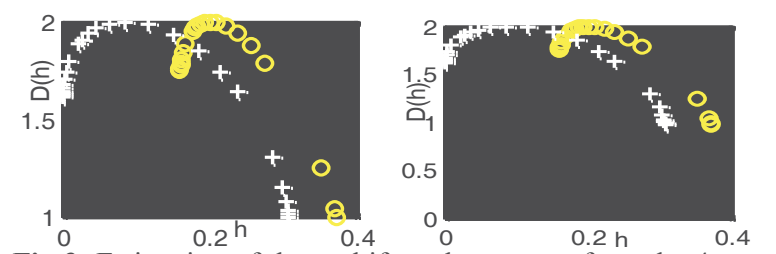

Fig 3. Estimation of the multifractal spectrum from the 4 samples of figure 2 .

The extracted features $\left(h_{q}\right)$ from our approach represent the degree of singularities of the underlying tissue for order $q$. $h_{0}$ is the most common degree of irregularity.

Monofractal parameters $(H, S, v a r)$ have been extracted from the diffusion equation [3]; $H$ is the Hurst parameter;

Var represents the saturation of the variance; and $S$, the characteristic size of the autosimilar element in the image.

\begin{tabular}{|c|c|c|c|c|c|c|}
\hline \multicolumn{6}{|c|}{ Multifractal formalism } & \multirow{2}{*}{$\begin{array}{c}\text { Fractal } \\
(H, S, \text { Var }) \\
\end{array}$} \\
\hline & $h_{2}$ & $h_{1}$ & $h_{0}$ & $h_{-1}$ & $h_{-2}$ & \\
\hline Melanomal & 0.2225 & 0.2102 & 0.2007 & 0.1933 & 0.1876 & \begin{tabular}{ll|l|}
$0.72|1.60| 4.39$ \\
\end{tabular} \\
\hline Melanoma2 & 0.4341 & 0.3911 & 0.3569 & 0.3239 & 0.2859 & $0.70|1.79| 3.31$ \\
\hline Melanoma3 & 0.4530 & 0.4185 & 0.3900 & 0.3676 & 0.3494 & $0.74|1.38| 4.45$ \\
\hline Melanoma4 & 0.4331 & 0.4366 & 0.4462 & 0.4484 & 0.4356 & $0.60|1.94| 3.48$ \\
\hline Melanoma5 & 0.3486 & 0.3236 & 0.3025 & 0.2840 & 0.2671 & \begin{tabular}{|l|l|}
$0.66|1.60| 3.51$ \\
\end{tabular} \\
\hline Melanoma6 & 0.2564 & 0.2344 & 0.2133 & 0.1939 & 0.1763 & $0.84|1.09| 3.58$ \\
\hline Melanoma7 & 0.5302 & 0.4385 & 0.3718 & 0.3275 & 0.2961 & $0.71|4.27| 3.27$ \\
\hline
\end{tabular}

Table2. Estimation of multifractal features form melanoma samples.

Tables 2 and 3 show the computed singularities at different orders for the melanoma and the skin, respectively. We can notice that values obtained, in the same image, from the melanoma and the normal skin are significantly different. This difference is particularly important for $h_{0}$. This can be explained by the higher complexity of scatters present in melanoma compared to normal skin [16]. In addition, the mean of $h_{q}$ for each tissue over the entire corpus remains different, with acceptable standard deviations (Table 4). 
We note that the multifractal approach gives better results. This preliminary result indicates the potential of our method to distinguish between different tissues.

\begin{tabular}{|c|c|c|c|c|c|c|}
\hline \multicolumn{6}{|c|}{ Multifractal formalism } & \multirow{2}{*}{$\begin{array}{c}\text { Fractal } \\
(H, S, \text { Var }) \\
\end{array}$} \\
\hline & $h_{2}$ & $h_{1}$ & $h_{0}$ & $h_{-1}$ & $h_{-2}$ & \\
\hline Skin 1 & 0.1507 & 0.1165 & 0.0868 & 0.0637 & 0.0472 & $0.66|1.60| 3.37$ \\
\hline Skin 2 & 0.1088 & 0.0971 & 0.0971 & 0.0751 & 0.0652 & $0.62|1.79| 3.38$ \\
\hline Skin 3 & 0.1625 & 0.1347 & 0.1104 & 0.0897 & 0.0725 & $0.76|1.38| 3.50$ \\
\hline Skin 4 & 0.1965 & 0.1784 & 0.2164 & 0.1377 & 0.1187 & $0.75|1.38| 3.37$ \\
\hline Skin 5 & 0.1490 & 0.1428 & 0.1359 & 0.1281 & 0.1195 & $0.70|1.60| 3.39$ \\
\hline Skin 6 & 0.2315 & 0.1344 & 0.0969 & 0.0638 & 0.0382 & $0.86|1.09| 3.47$ \\
\hline Skin 7 & 0.1001 & 0.0798 & 0.0608 & 0.0444 & 0.0315 & $0.93|1.09| 340$ \\
\hline
\end{tabular}

Table3. Estimation of multifractal features form normal skin samples

\begin{tabular}{|c|c|c|c|c|}
\hline & \multicolumn{3}{|c|}{ Multifractal formalism } & Fractal \\
\cline { 2 - 5 } & $h_{-2}$ & $h_{0}$ & $h_{2}$ & $H$ \\
\hline Skin & $0.0704 \pm 0.0335$ & $0.1149 \pm 0.0465$ & $0.1570 \pm 0.0428$ & $0.7543 \pm 0.1014$ \\
\hline Melanoma & $0.2683 \pm 0.0913$ & $0.3259 \pm 0.0850$ & $0.3826 \pm 0.1033$ & $0.7100 \pm 0.0682$ \\
\hline
\end{tabular}

Table4. Averages of multifractal features from the samples.

\section{CONCLUSION}

In this paper, we have investigated a preliminary study of the multifractality of the speckle in ultrasound images. We extracted meaningful parameters of a mutifractal spectrum for discrimination between ultrasound skin tissues annotated by dermatologists.

We proposed hierarchical multiresolution parameters based on the wavelet leaders. We have observed in practice that the leaders may consist of a large amount of estimated parameters. As an alternative, we have improved the computation by taking the same coefficient without the spatial neighborhood.

That led us to the definition of the maximum wavelet coefficients. The estimation of multifractal attributes of synthetic 2D process with known and controlled theoretical attributes showed that these coefficients are more efficient than the leaders, and moreover require less computation time.

We have applied this analysis to different samples of ultrasound skin images to extract a vector of features. This vector consists of singularities of order $q \in\{-2,-1,0,1,2\}$. The selected features discriminate a priori two groups (normal and pathological tissues),

We finally studied the variability of the features in the different regions (normal and melanoma) against the seven images and noticed that the estimated parameters are fairly stable.

We are currently conducting the validation of these parameters using large real medical data corpuses. We have also started investigating the feasibility of a segmentation method based on the proposed singularity features vector.

\section{REFERENCES}

[1] B.B. Mandelbrot, The fractal geometry of nature, W.H. freeman and company, New York, 1983.
[2] R. Lopes, N. Betrouni, "Fractal and multifractal analysis: A review," Medical Image Analysis, Elsevier, Vol. 13, pp. 634-649, 2009.

[3] S. Guyot, M-C. Peron and E. Deléchelle, "Spatial speckle characterization by Brownian motion analysis," Physical Review E, Statistical, nonlinear, and soft matter physics, vol. 70, Issue 4, pp. 046618.1-046618.8, 2004.

[4] D-R. Chen, R-F. Chang, C-J. Chen, M-F. Ho, S-J. Kuo, S-T. Chen, S-J Hung and W K. Moon, "Classification of breast ultrasound images using fractal feature," Journal of Clinical Imaging, Elsevier, Vol. 29, pp. 235-245, 2005.

[5] W-L. Lee, Y-C. Chen, Y-C Chen and K-S. Hsieh, "Ultrasonic liver tissues classification by fractal feature vector based on Mband wavelet transform," IEEE Transactions on Medical Imaging, Vol. 22, NO. 3, March 2003.

[6] W-L. Lee, Y-C. Chen, Y-C Chen and K-S. Hsieh, "Unsupervised segmentation of ultrasonic liver images by multiresolution fractal feature vector," Information Sciences, Elsevier, Vol. 175, pp. 177-199, 2005.

[7] M.H. Yap, E.A. Edirisinghe and H.E. Bez, "Object boundary detection in ultrasound images," IEEE computer society, Conference on Computer and Robot Vision, Canada, 2006.

[8] S. Jaffard, B. Lashermes , P. Abry, "Wavelet leaders in multifractal analysis", Wavelet Analysis and Applications, Birkhäuser Verlag, pp. 219-264, 2006.

[9] A. Arneodo, N. Decoster, and S. G. Roux, "A waveletbased method for multifractal image analysis. ie. methodology and test applications on isotropic and anisotropic random rough surfaces," European Physical Journal B 15, pp.567-600, 2000.

[10] H. Wendt, S.G. Roux, S. Jaffard and P. Abry, "Wavelet leaders and bootstrap for multifractal analysis of images," Signal Processing, Vol. 89, pp. 1100-1114, 2009.

[11] S. Mallat, "A wavelet tour of signal processing," Academic Press, San Diego, CA, 1998.

[13] A. Chhabra , C. Meneveau , R.V. Jensen and K.R. Sreenivasan, "Direct determination of the singularity spectrum and its application to fully developed turbulence'". Phys. Rev. A, 40(9), pp.5284 - 5294, 1989.

[12] M. Djeddi and A. Ouahabi, "Multifractal analysis based on the wavelet leaders applied to bone radiographic images," Master thesis, Center for Study and Research on Innovative Technologies for Disability and Health, University of Tours, 2009.

[14] E. Serrano and A. Figliola, "Wavelet leaders: A new method to estimate the multifractal singularity spectra,". Phys. Rev. A 388 , pp. $2793-2805,2009$.

[15] B.B. Mandelbrot,'Intermittent turbulence in selfsimilar cascades: divergence of high moments and dimension of the carrier," J. Fluid Mech, Vol. 62, pp. 331-358, 1974.

[16] M. Molski and J. Konarski, "Tumor growth in the space-time with temporal fractal dimension," Chaos, Solitons and Fractals, Elsevier, Vol. 36, pp. 811-818, 2008. 